\title{
Tabela de medidas para o desenvolvimento de vestuário para mulheres gestantes: uma proposição empírica
}

\author{
Measurements for the development \\ of clothing for pregnant women: \\ an empirical proposition
}

Fabiane Fischer Murara ${ }^{[1]}$, Mariana Luísa Schaeffer Brilhante $^{[2]}$, Vivian Andreatta $\operatorname{Los}^{[3]}$, Valdecir Babinski Júnior $^{[4]}$, Fernanda Cristina Fontana ${ }^{[5]}$, Isadora Rodolfo da Silva[6], Izabela Schwitzki ${ }^{[7]}$, Maria Eduarda Alves da Silva $^{[8]}$, Nicolly Colaço Pereira ${ }^{[9]}$

[1] Graduada em Moda, CATÓLICA SC. fabiane.fischer@ifsc.edu.br

[2] Mestranda em Design de Vestuário e Moda, UDESC. marsbxx@gmail.com

[3] Mestra em Educação, FURB. vlos@ifsc.edu.br

[4] Mestre em Design de Vestuário e Moda. UDESC.vj.babinski@gmail.com

[5] Estudante do Curso Técnico Integrado em Modelagem do Vestuário, IFSC. fernandafontana02@gmail.com

[6] Estudante do Curso Técnico Integrado em Modelagem do Vestuário, IFSC. isadora.giovanna01@gmail.com

[7] Estudante do Curso Técnico Integrado em Modelagem do Vestuário, IFSC.izabela.s@aluno.ifsc.edu.br

[8] Estudante do Curso Técnico Integrado em Modelagem do Vestuário, IFSC. mariaasduda101@gmail.com

[9] Estudante do Curso Técnico Integrado em Modelagem do Vestuário, IFSC.nihcp18@gmail.com 
Resumo: o presente artigo teve como objetivo propor um modelo de tabela de medidas que possa servir para o desenvolvimento de peças do vestuário para gestantes. A abordagem utilizada foi pautada em um levantamento bibliográfico, a partir de uma perspectiva voltada ao modo como são construídas as tabelas de medidas em vigor, e pelo mercado de vestuário, de forma que represente o público gestante. Com base na pesquisa realizada, concluiu-se que: (I) as medidas aferidas de gestantes do $1^{\circ}$ ao $6^{\circ}$ mês variam de acordo com seu tipo corpóreo, por exemplo, podem aumentar de dois a dez centímetros na circunferência do busto; e (II) não existem muitos estudos científicos e tabelas medidas assertivas para todos os biótipos corporais nesse segmento do ramo de vestuário.

Palavras-chaves: tabela de medidas. Gestantes. Antropometria. Vestuário.

Abstract: This article aimed to propose a model of measurement table that can be used for the development of garments for pregnant women. The approach used was based on a bibliographic survey, from a perspective focused on how the measurement tables in force are constructed, and by the clothing market, in a way that represents the pregnant public. Based on the research carried out, it was concluded that: (I) the measurements taken by pregnant women from the 1st to the 6th month vary according to their body type, for example, they can increase from two to ten centimeters in the circumference of the bust; and (II) there are not many scientific studies and assertive measurement tables for all body biotypes in this segment of the clothing industry.

Keywords: Measurement table. Pregnant women. Anthropometry. Clothing.

\section{INTRODUÇÃo}

De acordo com Stefani (2005), a palavra moda teve origem no latim modus, que significa modo ou maneira, a forma pela qual as pessoas se vestem. Já para Frings (2012), a moda consiste em um reflexo das forças sociais, políticas, econômicas e artísticas de um determinado período. Contemporaneamente, o termo emprega diversas interpretações. Indivíduos e seus grupos têm seus próprios conceitos a respeito do tema, que tem ampliado seus aspectos socioculturais em relação à idealização do corpo. Conforme Kehl e Bucci (2004, p. 176), para “[...] milhares de brasileiros, incentivados pela publicidade [...], o sentido da vida reduziu-se à produção de um corpo. A possibilidade de 'inventar' um corpo ideal [...]". A discriminação desse corpo inatingível recai diretamente sobre as gestantes e puérperas - mulheres no período do pós-parto, que dura a partir da saída da placenta até 45 dias após o nascimento da criança - uma vez que há dificuldades em internalizar e incorporar as mudanças na imagem corporal durante a gravidez. 
Durante a gestação, com relação ao vestuário, o que mais importa é o conforto das peças. De acordo com Machado (2014), ocorrem modificações físicas notáveis, que podem ser o aumento do quadril, dos seios e da barriga. Portanto, o autor considera indispensável a mobilidade na modelagem e nos tecidos, a segurança e o aconchego para os seios e a barriga, além das demais partes do corpo.

Igualmente, Machado (2014) destaca a relevância da ergonomia nesse cenário; pode-se dizer, então, que a ergonomia considera o ser humano e suas habilidades, limitações e características, torna o corpo humano o ponto de partida e respeita suas singularidades. Logo, as tabelas de medidas para a criação do vestuário devem seguir esse critério: o respeito às particularidades presentes no corpo das gestantes. "A tabela de medidas serve como base ao desenvolvimento de uma modelagem e é usualmente composta por nomenclaturas de tamanhos [...] e medidas do corpo humano [...]" (DINIS; VASCONCELOS, 2014, p. 72).

No mercado de gestantes, cada loja ou empresa possui sua própria tabela de medidas. Essas tabelas apresentam, normalmente, apenas a medida de circunferência do busto e do quadril. Essas medidas costumam divergir muito entre as marcas. Isso prejudica a possibilidade de uma padronização de informações (MACHADO, 2014). Machado (2014) ainda afirma que, atualmente, no Brasil, não existe uma tabela de medidas que possa ser considerada um padrão ou que represente as medidas da população brasileira. Portanto, fica a critério de cada empresa estabelecer sua tabela. Essa ausência de padronização traz dificuldades ao modelista. Ao fazer um comparativo entre as normas técnicas - como as da Associação Brasileira de Normas Técnicas (ABNT) ou por meio das bibliografias já existentes - e as contribuições científicas sobre o tema para a definição de qual tabela de medidas usar, aparecem divergências consideráveis entre as tabelas oferecidas no mercado, seja no uso de nomenclaturas, tamanhos, termos técnicos que definem medidas, partes do corpo mensuradas, entre outras (MACHADO, 2014). 
O período gestacional pode ser dividido de duas formas: (I) em trimestres, do primeiro ao terceiro mês, do quarto ao sexto e do sétimo ao nono mês de gestação, no qual o abdome da gestante cresce significativamente; ou (II) em meses, do primeiro até o nono. Nesses períodos, existe a necessidade de uma roupa ergonomicamente correta para cada fase. As primeiras mudanças do corpo acontecem na fase do primeiro até o sexto mês gestacional, e essa fase requer um tipo de vestuário com adequações que refletem em fatores físicos, sensoriais e motores. Afinal, o corpo está sujeito a alguns hormônios, como a progesterona - responsável pela conversão do endométrio - o que pode levar a alterações no estômago, cólon, bexiga, ureteres, vasos sanguíneos, entre outros.

Portanto, diante do exposto, o presente artigo buscou propor um modelo de tabela de medidas que possa servir para o desenvolvimento de peças do vestuário para gestantes. Visto que, atualmente, as poucas empresas que oferecem roupas para esse público constroem suas próprias tabelas de medidas. A ABNT oferece guias de parâmetros de medidas para diferentes tipos corporais:

Fornece uma descrição das medidas antropométricas que podem ser utilizadas como base para comparação de grupos populacionais. A lista básica especificada destina-se a servir como um guia para os ergonomistas quanto à definição de grupos populacionais e quanto à aplicação de seus conhecimentos para o projeto geométrico dos locais onde as pessoas trabalham e vivem. Não se destina a servir de um guia sobre como levantar medidas antropométricas, mas fornece informações aos ergonomistas e projetistas sobre as bases anatômicas e antropométricas e os princípios das medidas aplicados na solução de problemas projetuais (ASSOCIAÇÃO BRASILEIRA DE NORMAS TÉCNICAS, 2018, n.p).

Ao pesquisar essa NBR, infere-se que a Associação Brasileira de Normas Técnicas (2018) dispõe os métodos de aferição das principais medidas do corpo humano, mas, nas normas, não há nenhuma tabela de medidas específica para o público gestante, somente para o público feminino. Então, 
ressalta-se a importância de uma tabela de medidas para o público gestacional em literatura, visto que, atualmente, no mercado, cada loja e empresa constrói a sua própria e, por isso, majoritariamente, elas se divergem.

O estudo da pesquisa atual foi construído dentro das cinco perspectivas metodológicas de Gil (2008), a saber: (I) pesquisa aplicada, vista a realização de um protótipo final; (II) abordagem qualitativa; (III) perspectiva descritiva; (IV) revisão bibliográfica narrativa e assistemática; e (V) pesquisa de campo. Esse artigo está estruturado com base em uma fundamentação teórica sobre tabelas de medidas e modelagem. Após explorada a literatura da área, procede-se para os aspectos metodológicos, a descrição dos resultados e as considerações finais.

\section{TABELA DE MEDIDAS NO BRASIL}

A aferição de medidas corporais tornou-se necessária para a arte da alfaiataria, que consistia na elaboração de roupas alinhadas ao corpo e com a pretensão de velar determinadas imperfeições físicas dos usuários (JONES, 2005). Com o passar do tempo, já no século XIX, a arte da alfaiataria tornou-se uma ciência, afirmam Sabrá et al. (2013), onde passou-se a catalogar e medir os mais diferentes tipos de corpos humanos. O procedimento de medição era realizado com o uso de fitas, que mediam as larguras do corpo.

A tabela de medidas é um conjunto de medidas usadas como base na construção de peças de vestuário. Essa tabela tem seus valores baseados em medidas aferidas de um certo número de pessoas e é separada pelos tamanhos em grupos, que são representados por $\mathrm{P}$ (pequeno), $\mathrm{M}$ (médio) ou $\mathrm{G}$ (grande), e variações, como PP, GG, XG e outros para tamanhos menores ou maiores, podendo ir do 36 ao 54. Dinis e Vasconcelos (2014) ressaltam como é importante que cada empresa siga os padrões de uma tabela de medidas e que tenha um "corpo ou modelo de prova", normalmente sendo uma pessoa e/ou manequim que esteja dentro das medidas da tabela referente ao público que está sendo trabalhado pela empresa. 
De acordo Schimitt e Bataglion (2017), o corpo deve estar posicionado em uma forma padrão, sendo ela com braços paralelos ao corpo, com as palmas das mãos para a frente, postura ereta, pés no chão levemente afastados e calcanhares encostados. Essa posição é chamada de "posição ortostática". As medidas são tiradas do corpo de frente, perfil, costas e meio perfil. Cada tabela apresentará medidas de diferentes partes do corpo e de distintas formas, isso depende, igualmente, do tipo de peça que será fabricada, mas, de uma forma geral, as medidas presentes em tabelas são: altura do corpo, circunferência do busto e da cintura, largura do ombro e das costas, entre cavas e pescoço, para a parte superior do corpo; e circunferência do quadril, gancho, altura do joelho e tornozelo, para os membros inferiores. Quanto aos métodos e ferramentas de obtenção dessas medidas, eles são variados, alguns exemplos são: antropômetro, compasso curvo ou compasso de espessura, fita métrica flexível, entre outros.

Associação Brasileira de Normas Técnicas (2010), por meio da NBR ISO 7250-1, ressalta a importância de usar a fita métrica para a aferição de medidas de circunferências e alturas, e prevê que sejam mensuradas, por meio do aparelho chamado antropômetro, a massa corporal, a estatura e altura dos ombros e dos joelhos. Já a NBR ISO 9241-171 (ASSOCIAÇÃO BRASILEIRA DE NORMAS TÉCNICAS, 2018) traz diretrizes ergonômicas para o uso de aparelhos eletrônicos, móveis etc., mas não faz nenhuma referência à ergonomia do vestuário.

Com o avanço da tecnologia, a Indústria 4.0 trouxe novos resultados para a indústria da moda. Tornou-se possível realizar um estudo antropométrico de inferência populacional. Sabrá et al. (2013) e Longhi e Santos (2017) constatam que o instrumento de medição corporal mais adequado no mercado é o body scanner, pois trata-se de uma tecnologia de escaneamento a laser que projeta, por meio de luzes, listras finas e definidas no corpo da pessoa ou manequim, e a medição é feita por meio de regras geométricas. Essa luz é um feixe luminoso que utiliza lasers incapazes de causar mal à retina humana: são os eye-safe lasers. 
O IFSC campus Jaraguá do Sul/Centro trabalha com uma metodologia específica e própria de modelagem, chamada de "modelagem cartesiana", a qual utiliza o plano cartesiano como passo inicial para a construção das modelagens (THEIS, 2018). Essas regras, adotadas para a aferição das medidas corporais, divergem em alguns pontos se comparadas com outros autores, pois, dessa forma, as medidas de alturas passam a contar do ponto 0 do plano cartesiano, sendo esse localizado no pescoço do corpo humano, como no caso da altura da cintura e da altura do busto.

Denota-se que no IFSC campus Jaraguá do Sul/Centro a medida da largura das costas é obtida a partir das rótulas direita e esquerda dos braços; a medida da circunferência do busto é obtida na linha horizontal sobre os mamilos e a altura do busto pode ser aferida a partir da base do pescoço até o mamilo. Já a distância entre os seios parte do mamilo direito até o esquerdo, e a medida do raio dos seios é encontrada a partir do centro do mamilo até a base inferior do seio. Para encontrar a altura da cintura parte-se da base do pescoço até a cintura, que se localiza logo ao fim da última costela, onde a circunferência da cintura é obtida (linha horizontal do corpo, logo abaixo da última costela). Também se obtém a circunferência da barriga na parte mais saliente desta, próxima à região do umbigo, e a circunferência do quadril é obtida na linha horizontal do tronco, onde se encontra o maior volume, na parte mais saliente das nádegas (THEIS, 2018).

Sabrá et al. (2013) também trazem informações sobre softwares que fazem a leitura das medidas do corpo por meio de fotos, onde raios de laser, inofensivos aos seres humanos, dimensionam o corpo em circunferências e alturas. Ainda assim, os autores apontam para os custos elevados desses softwares e para as desvantagens quanto à sensibilidade do software aos movimentos involuntários do indivíduo. Afinal, é praticamente impossível permanecer imóvel por muitos segundos, e movimentos involuntários como respirar ou contrair músculos podem gerar erros na medição. 
Segundo Soares e Petroski (2003), a antropometria é uma palavra de origem grega, composta de "antropo", "homem", e "metria" - que significa medida. A antropometria é a ciência das medidas humanas, ela aborda a estrutura mecânica e comportamento dos materiais biológicos. Para Boueri (2010, p. 63) a antropometria compreende as dimensões, composição e propriedade de massa dos segmentos do corpo; ligados às ações voluntárias do corpo em relação ao controle dos movimentos, na "aplicação de forças, torções, energia e potência", em relação a objetos externos, controles, ferramentas e outros equipamentos, ou seja, é responsável por proporcionar o conforto da peça ao usuário.

Igualmente, pode ser considerado o ponto de partida para a elaboração efetiva de uma tabela base de medidas para a produção das roupas. Esses dados variam em cada país ou região, pois as medidas mudam de um lugar para outro. Silveira $(2008 ; 2017)$ relata que as medidas do corpo dependem de variáveis como faixa etária, biotipo, gênero, clima, alimentação e saúde. Esses dados podem ser mensurados por meio da tecnologia do body scanner, desenvolvida pela empresa criada em 1979, TC$^{2}$, que projeta o corpo e torna possível a análise de suas medidas e padrões. Longhi e Santos $(2017$, p. 1) encontraram “[...] um estudo recente, realizado em nível nacional, que confirmou a existência de sete biótipos na população feminina brasileira". Os autores afirmam, também, que essa sistematização de dados antropométricos por meio da Tabela de Medidas Associáveis, a chamada TMA, que é um método de sistematização de medidas desenvolvido pelos autores, onde as medidas obtidas são reunidas por sete tipos de algoritmos, que podem ser usados simultaneamente na construção de tabelas de medidas. De acordo com Longhi e Santos (2017), a TMA permite ao modelista trabalhar com os dados de mais de um biotipo simultaneamente, uma vez que apresenta medidas dos sete biótipos existentes ${ }^{[10]}$. Também permite utilizar comprimentos variados e acrescentar dados de outros biotipos.
[10] Os tipos de biotipo existente, segundo Longhi e Santos (2017) e Lee et al. (2007), são: (B1) biótipo ampulheta; (B2) biótipo ampulheta inferior; (B3) biótipo ampulheta superior; (B4) biótipo colher; (B5) biótipo triângulo; (B6) biótipo triângulo invertido; e (B7) biótipo retângulo. 
Boueri (2008) argumenta que o corpo pode ser estudado como suporte do produto na área do vestuário, e mais estudos sobre suas medidas e padrões devem ser realizados. Para o projeto do vestuário, faz-se necessário o conhecimento do perfil antropométrico do usuário e a elaboração de tabelas de medidas adequadas ao público. Com o uso de técnicas de modelagem ergonomicamente adaptadas, consegue-se atender bem ao público gestante e todos os gêneros, sexos, etnias e tipos corpóreos.

Quanto ao exposto, a ABNT, no ano de 1995, divulgou a NBR 13377 - Medidas do corpo humano para vestuário - feminino, masculino e infantil. Ela quase não foi utilizada para tentar padronizar os tamanhos de peças do vestuário, pois não atendia às exigências dos consumidores, afinal, cada consumidor tem uma necessidade diferente e cada etnia possui padrões corporais diferentes. Essa norma foi inutilizada no ano de 2012 e, atualmente, a ABNT trabalha em novas tabelas de medidas para o público feminino, sendo que, para os públicos masculino e infantil, já foram criadas tabelas que estão em uso.

Em caráter de curiosidade, aborda-se que a ABNT também fornece normas técnicas de padronização nos EUA, por meio de sua marca, a ASTM (ASTM INTERNACIONAL, 2021). Antes dos padrões ASTM 4 - a última geração - serem desenvolvidos, uma progressão aritmética de duas polegadas para cinco era usada entre todos os tamanhos das tabelas de medidas, para criar as diferenças entre os tamanhos. Os fabricantes perceberam que era necessário utilizar uma diferença de uma polegada entre tamanhos menores, uma polegada e meia entre os tamanhos 10 e 12 e duas polegadas entre os tamanhos maiores que 12. Algumas empresas estadunidenses seguem os padrões ASTM (ASTM INTERNACIONAL, 2021), porém fabricantes, consumidores e varejistas relatam que esses padrões não condizem com as medidas reais. 


\section{TABELA DE MEDIDAS PARA GESTANTES}

\section{E A ERGONOMIA NO VESTUÁRIO}

De acordo com Browne et al. (1949), a ergonomia é o estudo do relacionamento entre o homem e seu trabalho junto à aplicação dos conhecimentos de anatomia, fisiologia e psicologia na solução dos problemas surgidos desse relacionamento. Já para Wisner (1987), é o conjunto dos conhecimentos científicos relacionados ao homem e necessários à concepção de instrumentos, máquinas e dispositivos para que possam ser utilizados com o máximo de conforto, segurança e eficiência. Então, nota-se como ergonomia é um passo fundamental para a construção de tabelas de medidas e da modelagem em si, pois, como afirma Martins (2005), para a elaboração de produtos é necessário ter em mente as necessidades psicológicas, físicas, psicofisiológicas e antropométricas do usuário, fundamentalmente para um período de tanta mudança em todos os aspectos, como é a gestação. Segundo Machado (2014, p. 20):

[...] a gestante perde um pouco da cintura, o quadril, seio e barriga aumentam de tamanho. É preciso que o produto traga conforto, segurança, aconchego principalmente para a barriga e seios, quadril e outras partes do corpo. O padrão de roupas para este público deve ser antropometricamente e ergonomicamente pensados para o seu conforto e para que isso seja possível necessitase de tabelas de medidas adequadas a este público.

No Brasil, algumas tabelas de medidas são construídas pela ABNT ou pelas próprias empresas de confecção de vestuário, como é o caso da Radicetti (1999) —empresária responsável pela empresa de manequins Draft - que, ao realizar pesquisas de campo com seu público-alvo, por meio do uso de modelos que provam suas peças pilotos, também chamadas de "corpo de prova", procuram aumentar a credibilidade e a assertividade nos produtos que oferecem ao mercado, sejam eles roupas ou manequins de moulage. 
Não há regras claras a serem seguidas quanto à aferição de medidas, mas há algumas diretrizes na NBR ISO 7250-1 (ASSOCIAÇÃO BRASILEIRA DE NORMAS TÉCNICAS, 2010) para esse trabalho, porém, na ABNT, essas sugestões de normas não se referem ao público gestante. Corroborando ao exposto, Dinis e Vasconcelos (2014) afirmam que,

[...] atualmente, o Brasil não possui uma tabela de medidas que possa ser considerada "padrão representativo" da população brasileira, ficando a critério de cada empresa o estabelecimento e utilização de sua própria tabela. Muitos profissionais da área de modelagem recorrem às normas técnicas, como as da ABNT ou a bibliografias existentes para fazer um comparativo entre elas. Nisso, eles se deparam com um dos problemas mais corriqueiros: a ausência de padronização, seja no uso de nomenclaturas de tamanhos, nos termos técnicos para definir as medidas (ex. em fontes bibliográficas diferentes, podem-se encontrar os termos manga e braço para definir a medida do comprimento do braço), na metodologia de medição manual e nas próprias medidas matemáticas (DINIS; VASCONCELOS, 2014, p. 72).

As próprias gestantes, ao realizarem a compra de vestuário de marcas diferentes, seja por maneira física ou por e-commerce, sentem dificuldades de entender qual tamanho usam, afinal, as medidas dos tamanhos podem variar entre marcas. Segundo Monteiro e Carvalho (2018, n.p), ao abordar o assunto compras on-line, "[...] as clientes tendem a ficar confusas quando efetuam uma compra em que as informações da grade de medidas são imprecisas sobre o tamanho do produto, já que não há um valor de referência estipulado pela ABNT". Sem uma tabela de medidas adequada aos padrões corpóreos da população brasileira e das gestantes torna-se difícil a compra pela internet. Existem modelagens e tipos de peças do vestuário que se tornam ainda mais difíceis de usar ao longo da gravidez, principalmente pela inexistência de tabelas de medidas assertivas para esse público. Diante de tais questões, estuda-se, de maneira comparativa, algumas tabelas de medidas para gestantes, apresentadas a seguir.

$\mathrm{F}$ 


\section{(ษ) Batas, Blusas e Vestidos}

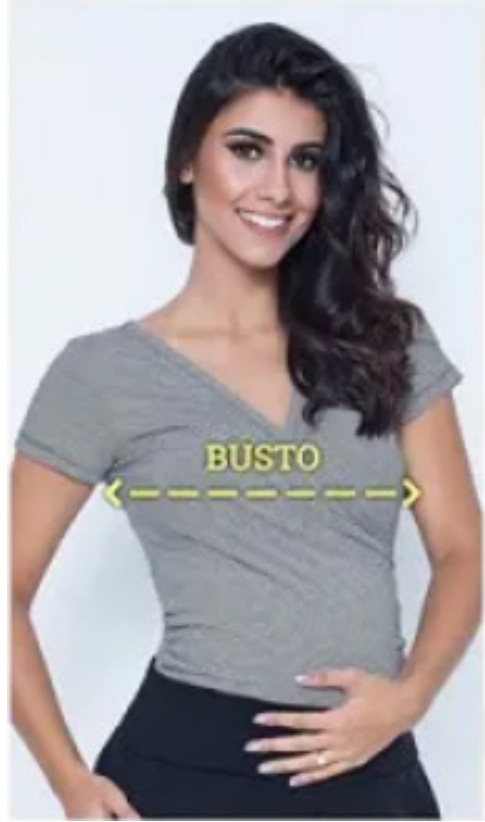

$\begin{array}{cc}\text { Medida } & \text { Tamanho } \\ \text { do Busto } & \text { A Gestante }\end{array}$

$81 \mathrm{~cm} \cdot 87 \mathrm{~cm}$

$88 \mathrm{~cm}-94 \mathrm{~cm}$

$95 \mathrm{~cm} \cdot 101 \mathrm{~cm}$

$102 \mathrm{~cm}-108 \mathrm{~cm}$

$109 \mathrm{~cm} \cdot 115 \mathrm{~cm}$

$116 \mathrm{~cm}-121 \mathrm{~cm}$

\section{Comprimento Vestido Curto}

$126 \mathrm{~cm}$
PP

P

M

G

GG

EXG

(n) Calças, Bermudas e Macacões

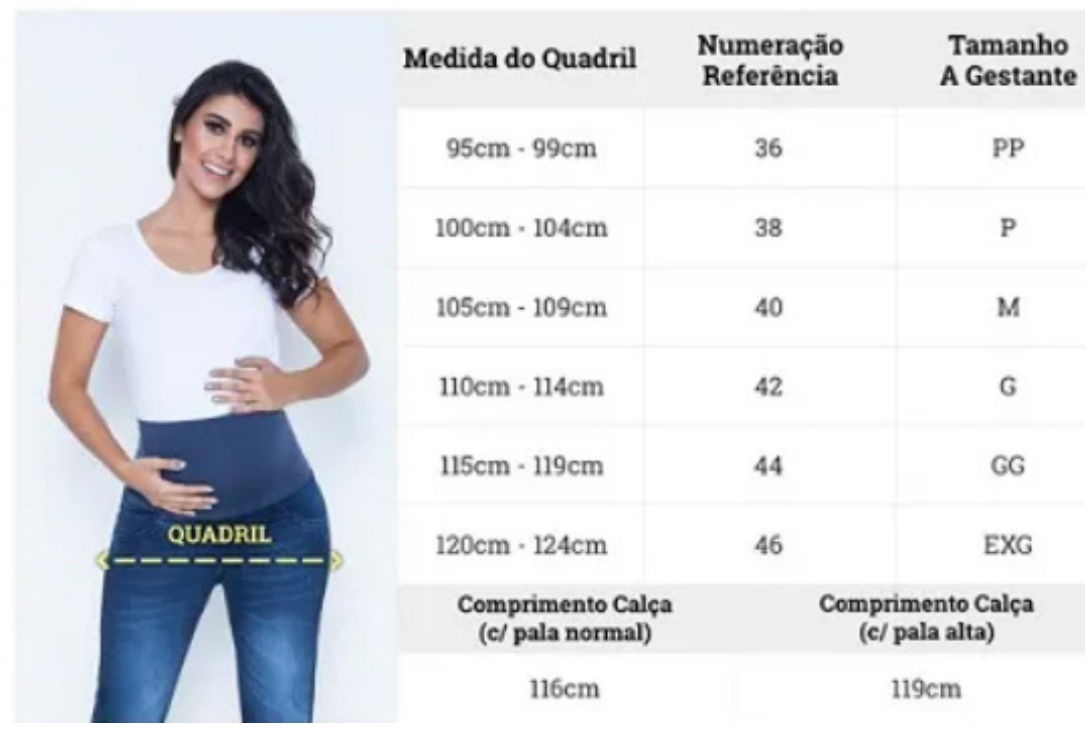

Figura 1 - Tabela de medidas para o público gestacional da loja "A Gestante". Fonte: https://www. agestante.com.br/.

Nas pesquisas realizadas para esse artigo, percebe-se que a ABNT utiliza as dimensões da cintura, mesmo essa tabela sendo feita para peças femininas bottom - "partes de baixo" - , como calças e saias, pois a NBR 13377 (ASSOCIA- 
ÇÃO BRASILEIRA DE NORMAS TÉCNICAS, 1995) afirma que as medidas da cintura são usadas majoritariamente como referência, e servem para a construção dessas peças (OLIVETE, 2020). Já nas tabelas disponíveis em lojas e/ou marcas de roupas para gestantes, as peças de roupas como calças, shorts e saias são confeccionadas com base na medida da circunferência do quadril, e não da cintura, pois, conforme Machado (2014), essa medida sofre muitas alterações e passa a ser substituída pela medida da circunferência da barriga, que se torna maior. Porém, conforme a análise das tabelas de medidas aqui apresentadas, percebe-se que a maioria delas não demonstram a medida de circunferência da barriga.

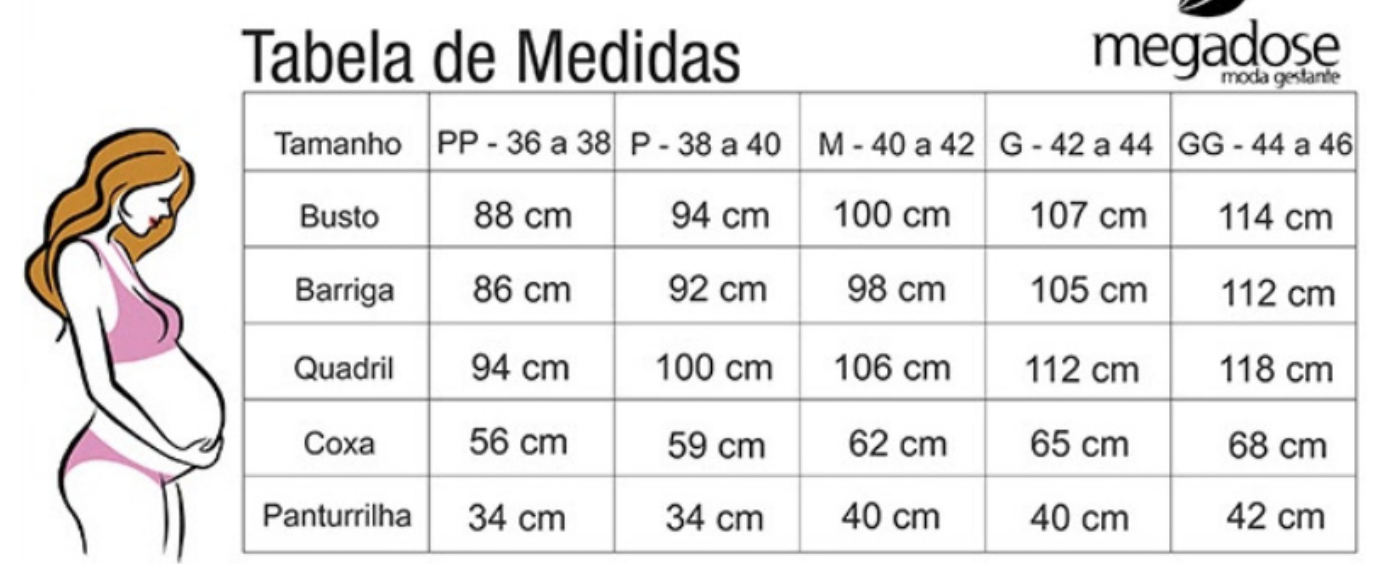

\section{Guia de Medidas}

Busto

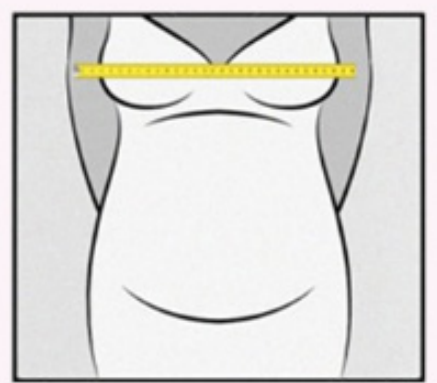

\begin{tabular}{|c|c|c|}
\hline \multicolumn{2}{|c|}{ Tamanhos } & Medidas Busto \\
\hline 40 & P & $88 \mathrm{~cm}$ a $94 \mathrm{~cm}$ \\
\hline 42 & M & $95 \mathrm{~cm}$ a $101 \mathrm{~cm}$ \\
\hline 44 & G & $102 \mathrm{~cm}$ a $108 \mathrm{~cm}$ \\
\hline 46 & GG & $109 \mathrm{~cm}$ a $115 \mathrm{~cm}$ \\
\hline 48 & XG & $116 \mathrm{~cm}$ a $121 \mathrm{~cm}$ \\
\hline
\end{tabular}

Medidas válidas para batas, blusas, regatas, camisas, camisolas e vestidos.

\section{Quadril}

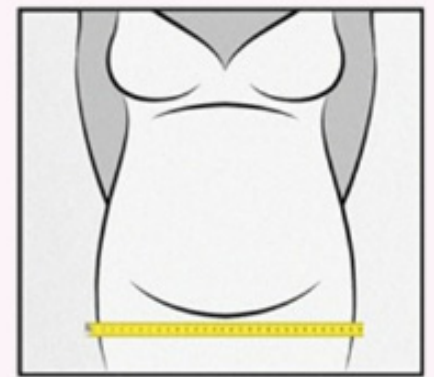

\begin{tabular}{|c|c|c|}
\hline \multicolumn{2}{|c|}{ Tamanhos } & Medidas Quadril \\
\hline 40 & P & $100 \mathrm{~cm}$ a $105 \mathrm{~cm}$ \\
\hline 42 & M & $104 \mathrm{~cm}$ a $109 \mathrm{~cm}$ \\
\hline 44 & $\mathrm{G}$ & $108 \mathrm{~cm}$ a $113 \mathrm{~cm}$ \\
\hline 46 & GG & $112 \mathrm{~cm}$ a $117 \mathrm{~cm}$ \\
\hline 48 & XG & $116 \mathrm{~cm}$ a $121 \mathrm{~cm}$ \\
\hline
\end{tabular}

Medidas válidas para calças, macacões, bermudas, shorts e pijamas.
Figura 2 - Tabela de medidas para o público gestacional da loja "Megadose".

Fonte: https://megadose.com.br/
Figura 3 - Tabela de medidas para o público gestacional da loja "Gestante Fashion". Fonte: https://www. gestantefashion.com.br/

\section{Evite Trocas}

Analise o tamanho das roupas conforme esta tabela. 

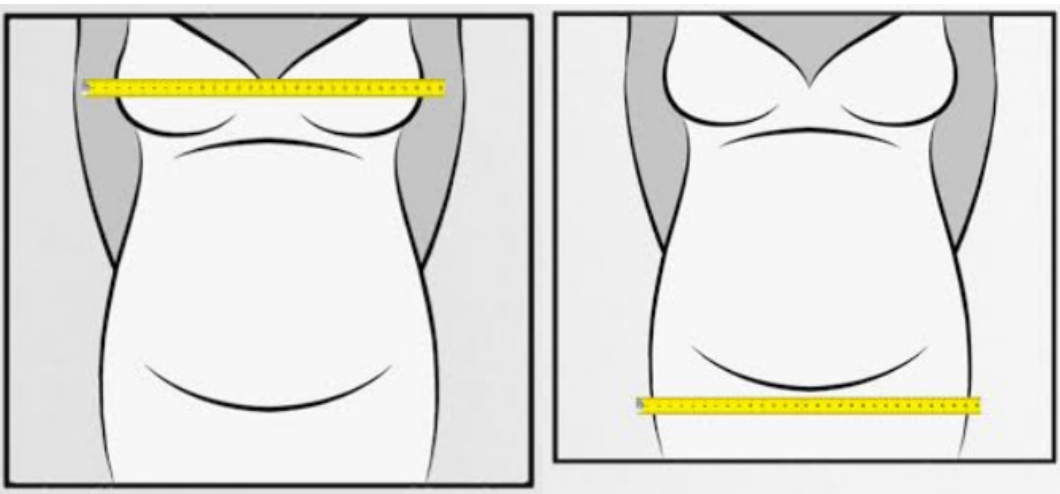

\begin{tabular}{|c|c|c|}
\hline \multicolumn{2}{|c|}{ TAMANHOS } & MEDIDA - BUSTO \\
\hline 40 & $\mathrm{P}$ & $88 \mathrm{~cm}$ a $94 \mathrm{~cm}$ \\
\hline 42 & M & $95 \mathrm{~cm}$ a $101 \mathrm{~cm}$ \\
\hline 44 & G & $102 \mathrm{~cm}$ a $108 \mathrm{~cm}$ \\
\hline 46 & GG & $109 \mathrm{~cm}$ a $115 \mathrm{~cm}$ \\
\hline
\end{tabular}

\begin{tabular}{|c|c|c|}
\hline \multicolumn{2}{|c|}{ TAMANHOS } & MEDIDA - QUADRIL \\
\hline 40 & $\mathrm{P}$ & $100 \mathrm{~cm}$ a $105 \mathrm{~cm}$ \\
\hline 42 & $\mathrm{M}$ & $104 \mathrm{~cm}$ a $109 \mathrm{~cm}$ \\
\hline 44 & $\mathrm{G}$ & $108 \mathrm{~cm}$ a $113 \mathrm{~cm}$ \\
\hline 46 & GG & $112 \mathrm{~cm}$ a $117 \mathrm{~cm}$ \\
\hline 48 & EXG & $116 \mathrm{~cm}$ a $121 \mathrm{~cm}$ \\
\hline 50 & EXGG & $120 \mathrm{~cm}$ a $125 \mathrm{~cm}$ \\
\hline
\end{tabular}

Figura 4 - Tabela de medidas para o público gestacional da loja "Gestante \& Cia". Fonte: https://www. lojagestanteecia.com.br/

Nessas duas últimas tabelas apresentadas pode-se perceber que as medidas se repetem, porém as grades de tamanhos são diferentes, sendo que a marca "Gestante Fashion" oferece mais opções de tamanhos para as medidas de busto e a marca "Gestante \& Cia" oferece mais opções de tamanhos para as medidas de quadril. Ao analisar essas tabelas constata-se que, nas modificações físicas notáveis no corpo das gestantes, ocorre um aumento significativo nessas partes do corpo: quadril, seios e barriga (MACHADO, 2014).

Essas constatações fortalecem a visão que Dinis e Vasconcelos (2014) trazem ao afirmar que, no Brasil, não se há estudos que resultam na construção de tabelas de medidas que representam o padrão corpóreo brasileiro. Para o público gestante esses estudos se tornam ainda mais limitados. 


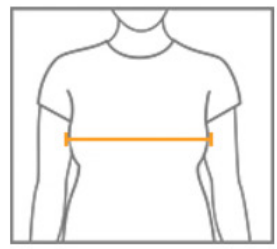

BUSTO

De pé ou

sentada, com

a coluna

ereta, meça a

altura de maior volume do seu

busto. Medidas válidas para:

Batas, Blusas, Camisas, Regatas e

Vestidos.

\begin{tabular}{|c|c|c|}
\hline \multicolumn{2}{|c|}{ TAMANHOS } & MEDIDAS - BUSTO \\
\hline 38 & PP & $81 \mathrm{~cm}$ a $87 \mathrm{~cm}$ \\
\hline 40 & P & $88 \mathrm{~cm}$ a $94 \mathrm{~cm}$ \\
\hline $42 / 44$ & M & $95 \mathrm{~cm}$ a $101 \mathrm{~cm}$ \\
\hline 46 & G & $102 \mathrm{~cm}$ a $108 \mathrm{~cm}$ \\
\hline 48 & GG & $109 \mathrm{~cm}$ a $115 \mathrm{~cm}$ \\
\hline
\end{tabular}

\section{QUADRIL}

De pé, junte as pernas e meça na altura de maior volume do seu quadril. Medidas válidas para: Calças,

Bermudas e Shorts, em jeans e sarja.

Figura 5 - Tabela de medidas para o público gestacional da loja "Belly Home". Fonte: https://www. bellyhome.com.br/

\section{PROCEDIMENTOS METODOLÓGICOS}

O atual artigo classifica-se como uma pesquisa qualitativa. Segundo Silveira e Córdova (2009, p. 32), “[...] a pesquisa qualitativa trata dos métodos que buscam explicar o porquê das coisas, exprimindo o que convém ser feito, mas sem quantificam os valores e as trocas simbólicas nem se submetem à prova de fatos, pois os dados analisados são não-métricos [...]". Por meio da pesquisa bibliográfica realizada buscou-se reunir autores selecionados de forma assistemática, para compreender se existem tabelas de medidas específicas para gestantes e, em caso afirmativo, se elas possuem caráter assertivo nas medidas.

Logo após a pesquisa bibliográfica realizou-se uma revisão de conceitos baseados nos autores abordados na pesquisa, uma pesquisa em sites de empresas de Jaraguá do Sul e região e, por fim, de marcas com vendas on-line que ofereciam tabelas de medidas para gestantes. Foram realizadas as análises dessas tabelas de medidas com base nos resultados obtidos com a pesquisa e com base na experiência profissional da docente Fabiane Fischer Murara. Nesse momento, partiu-se para uma pesquisa aplicada, que "[...] pode ser definida como atividades em que conhecimentos previamente adquiridos são utilizados para coletar, selecionar e processar fatos e dados, a fim de se obter e confirmar resultados, e sem gerar impacto" (FLEURY; WERLANG, 2016, p. 11-12). 
A coleta de dados e a proposição das tabelas de medidas foram resultado de um trabalho empírico que, conforme Gerhardt e Silveira (2009, p. 18), “[...] é o conhecimento que adquirimos no cotidiano, por meio de nossas experiências. É construído por meio de tentativas e erros num agrupamento de ideias. É caracterizado pelo senso comum, pela forma espontânea e direta de entendermos". A seguir apresenta-se o relato de experiência e as discussões oriundas da pesquisa.

\section{RELATO DE EXPERIÊNCIA E DISCUSSÕES}

Aborda-se um relato de experiência a fim de explicitar como foi construída a tabela de medidas para o público gestante. Construiu-se a tabela de modo empírico, mas com base nas experiências profissionais da docente Fabiane Fischer Murara, que ministra unidades curriculares voltadas a área de modelagem, ergonomia e construção de peças do vestuário.

A professora relata que, ao obter as medidas corporais de um considerável grupo de pessoas, é possível formular uma tabela de medidas. Porém, em escala industrial a aferição de medidas deve seguir determinados padrões, ou seja, regiões do corpo que devem ser mensuradas de acordo com um número expressivo de pessoas, visto que as empresas visam atender a um segmento de mercado para obter lucros, vender mais e servir de maneira atraente seus clientes. Diante disso, torna-se necessária a elaboração de tabelas de medidas que atendam a maior parte do seu público-alvo.

Para o público gestante verificou-se, por meio dos estudos de marcas ofertantes de roupas nesse segmento em Jaraguá do Sul e região, que não há uma grande oferta de vestuário para esse público. As tabelas de medida desse público-alvo, consequentemente, tornam-se escassas e com medidas que não atendem corretamente a todas as variações do corpo da gestante, principalmente no período do sexto ao nono mês de gestação. Verificou-se, também, a pouca quantidade de pesquisas científicas voltadas a essas questões. 
Essa pesquisa buscou propor uma tabela de medidas destinada ao público gestante no seu $6^{\circ}$ mês gestacional. Porém, como cada corpo gestante é um corpo que gera um outro corpo, muitas variações podem existir de uma gestante para outra. Com base nas medidas obtidas de gestantes ao longo de seis meses, foi realizada uma comparação entre as medidas corporais dessas gestantes e constatou-se o aumento significativo do início da gestação até o $6^{\circ}$ mês.

Da mesma forma, também foram utilizadas as medidas corporais dos manequins de modelagem da marca Draft, para realizar comparação entre o manequim feminino não gestante com o manequim feminino gestante no seu sexto mês gestacional. Nas imagens a seguir, percebem-se as diferenças entre os manequins femininos não gestantes e o manequim gestante. Nota-se o aumento de volume na região dos seios, quadril e, principalmente, cintura. As circunferências das coxas também aumentam em função de sustentar o peso da barriga.

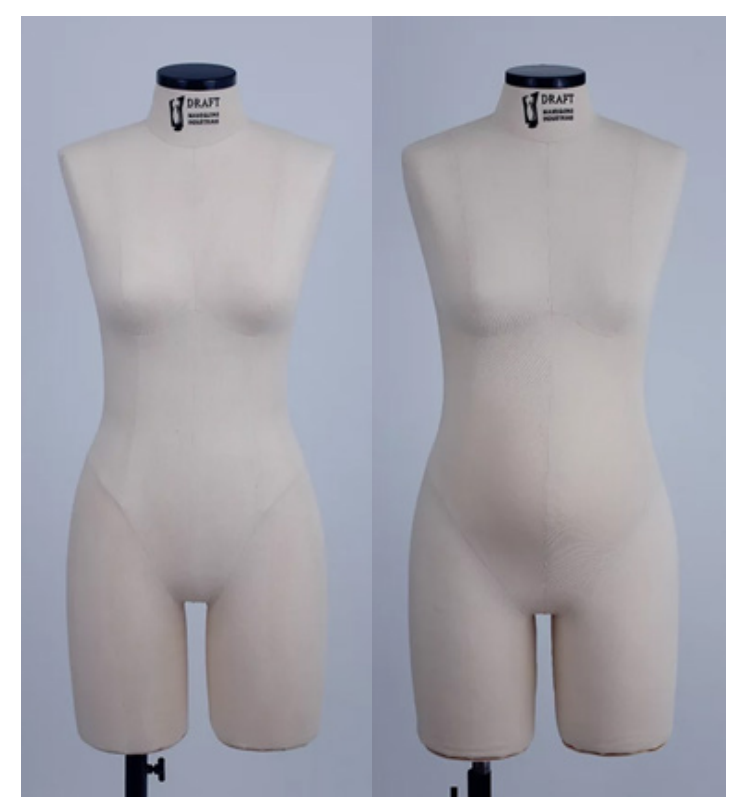

Figura 6 - Manequim feminino tamanho 40 e manequim gestante de 6 meses. Fonte: Site Draft Manequins (2021). 
Para esta pesquisa, inicialmente, foram aferidas as medidas dos manequins Draft. Posteriormente, junto ao grupo de estudantes e demais professores pesquisadores, aferiu-se as medidas das gestantes.

As medidas apuradas nos corpos das gestantes foram baseadas, principalmente, nas regiões do tronco superior até o quadril, visto que não haveria alterações referente a sua estatura. Porém, ao aferir as medidas no início da gestação e novamente no $6^{\circ}$ mês, percebeu-se que outras medidas também não se alteraram, como a largura das costas, entre outras medidas. Portanto, essa pesquisa focou nas medidas que são mais modificadas, como o busto, a barriga e o quadril, o que já denotava Machado (2014).

Para obter a aferição das principais medidas que sofreram aumento foi necessário o uso da fita métrica, de maneira a contornar o corpo com a mesma, para obter a medida na parte mais saliente do corpo nas regiões do busto, da barriga e do quadril (Quadro 1), conforme afirmava a pesquisa de Theis (2018).

Outro fator que precisa ser mencionado nessa pesquisa é que, diferente dos corpos femininos não gestantes, onde as medidas da região central do tronco se baseiam na cintura, no caso da gestante, a cintura passa despercebida, pois o maior volume corporal conta na região da barriga, próximo ao umbigo. Então, os resultados apresentados nessa pesquisa divergem das pesquisas de Dinis e Vasconcelos (2014) e da Associação Brasileira de Normas Técnicas (2004) quanto à aferição de medidas da cintura como padrão para algumas modelagens, visto que o que realmente importa é a medida da barriga em sua dimensão mais larga. 
Quadro 1 - Tabela de medidas de gestantes do $1^{\circ}$ ao $3^{\circ}$ mês

e dos manequins femininos Draft não gestantes

\begin{tabular}{|c|c|c|c|c|c|c|}
\hline Gestante A & Gestante B & Gestante C & Gestante D & Gestante E & $\begin{array}{c}\text { Manequim } \\
\text { tam. } 40\end{array}$ & $\begin{array}{c}\text { Manequim } \\
\operatorname{tam} .44\end{array}$ \\
\hline $98 \mathrm{~cm}$ & $95 \mathrm{~cm}$ & $84 \mathrm{~cm}$ & $82 \mathrm{~cm}$ & $80 \mathrm{~cm}$ & $88 \mathrm{~cm}$ & $96 \mathrm{~cm}$ \\
\hline $98 \mathrm{~cm}$ & $84 \mathrm{~cm}$ & $78 \mathrm{~cm}$ & $80 \mathrm{~cm}$ & $76 \mathrm{~cm}$ & $76 \mathrm{~cm}$ & $83 \mathrm{~cm}$ \\
\hline $110 \mathrm{~cm}$ & $108 \mathrm{~cm}$ & $93 \mathrm{~cm}$ & $94 \mathrm{~cm}$ & $90 \mathrm{~cm}$ & $98 \mathrm{~cm}$ & $106 \mathrm{~cm}$ \\
\hline
\end{tabular}

Fonte: desenvolvido pelos autores (2021).

Essa pesquisa, por se tratar de um experimento empírico, utilizou apenas a fita métrica como instrumento de aferição das medidas corporais, tanto para as medidas das gestantes como também dos manequins gestantes da marca Draft. Esse método de aferição seguiu as regras utilizadas pelos professores do IFSC campus Jaraguá do Sul/Centro, referenciadas pela dissertação de Theis (2018). E, por fim, realizou-se uma comparação entre as medidas aferidas das gestantes pesquisadas e comparadas com as medidas dos manequins Draft, o que trouxe padrões de aumento para cada parte do corpo gestante. Ao comparar as gestantes dos Quadros 1 e 2, é possível notar que a gestante $C$, por exemplo, aumentou cerca de dez centímetros na circunferência do busto. Já a gestante E, que já possuía uma estrutura de corpo menor, aumentou apenas dois centímetros nessa mesma circunferência.

Ao analisar a circunferência da barriga, constata-se que a gestante $B$ aumentou 18 centímetros, enquanto a gestante E aumentou apenas oito centímetros. Ao comparar a circunferência do quadril, percebe-se que a gestante $A$ aumentou 
sete centímetros na sua circunferência, já as gestantes $D$ e $E$ aumentaram quatro centímetros na circunferência de quadril. Entre os manequins gestantes da marca Draft citados na pesquisa, a medida da circunferência de busto do manequim tamanho 44 aumentou dez centímetros, a medida da circunferência da barriga aumentou onze centímetros e a circunferência do quadril aumentou oito centímetros. Já o manequim tamanho 40 teve aumentos menores do que o tamanho 44, o que também evidencia que estruturas de corpos maiores tendem a aumentar as medidas durante o período da gestação.

\section{Quadro 2 - Tabela de medidas de gestantes no $6^{\circ}$ mês gestacional}

\begin{tabular}{|c|c|c|c|c|c|c|}
\hline Gestante A & Gestante B & Gestante C & Gestante D & Gestante E & $\begin{array}{c}\text { Manequim } \\
\text { tam. } 40\end{array}$ & $\begin{array}{c}\text { Manequim } \\
\text { tam. } 44\end{array}$ \\
\hline $103 \mathrm{~cm}$ & $101 \mathrm{~cm}$ & $94 \mathrm{~cm}$ & $86 \mathrm{~cm}$ & $82 \mathrm{~cm}$ & $92 \mathrm{~cm}$ & $106 \mathrm{~cm}$ \\
\hline $114 \mathrm{~cm}$ & $102 \mathrm{~cm}$ & $94 \mathrm{~cm}$ & $90 \mathrm{~cm}$ & $84 \mathrm{~cm}$ & $90 \mathrm{~cm}$ & $94 \mathrm{~cm}$ \\
\hline $117 \mathrm{~cm}$ & $113 \mathrm{~cm}$ & $98 \mathrm{~cm}$ & $98 \mathrm{~cm}$ & $94 \mathrm{~cm}$ & $102 \mathrm{~cm}$ & $114 \mathrm{~cm}$ \\
\hline
\end{tabular}

Medida da

circunferência do busto

\section{Medida da} circunferência da barriga

Medida da circunferência do quadril

Com base nas análises dos Quadros 1 e 2 e na fundamentação teórica realizou-se a proposta de tabela de medida, que pode ser observada no Quadro 3.

É importante explicitar que essa é uma proposta de tabela de medidas destinada ao desenvolvimento de peças para gestantes do $6^{\circ}$ mês gestacional, construída com base nas medidas obtidas de algumas gestantes, como também dos manequins gestantes e não gestantes da marca Draft em comparação com tabelas de medidas das marcas apresentadas nesse estudo e, também, da forma de aferição dessas medidas. 
Quadro 3 - Proposta de tabela de medidas para gestantes no $6^{\circ}$ mês gestacional

\begin{tabular}{|c|c|c|c|c|c|c|c|c|}
\hline Tamanhos & 34 & 36 & 38 & 40 & 42 & 44 & 46 & 48 \\
\hline $\begin{array}{l}\text { Largura das } \\
\text { costas }\end{array}$ & $36 \mathrm{~cm}$ & $38 \mathrm{~cm}$ & $38 \mathrm{~cm}$ & $40 \mathrm{~cm}$ & $40 \mathrm{~cm}$ & $42 \mathrm{~cm}$ & $42 \mathrm{~cm}$ & $44 \mathrm{~cm}$ \\
\hline $\begin{array}{l}\text { Circunferência } \\
\text { do busto }\end{array}$ & $84 \mathrm{~cm}$ & $86 \mathrm{~cm}$ & $88 \mathrm{~cm}$ & $94 \mathrm{~cm}$ & $100 \mathrm{~cm}$ & $106 \mathrm{~cm}$ & $112 \mathrm{~cm}$ & $118 \mathrm{~cm}$ \\
\hline Altura do busto & $24 \mathrm{~cm}$ & $24 \mathrm{~cm}$ & $26 \mathrm{~cm}$ & $26 \mathrm{~cm}$ & $28 \mathrm{~cm}$ & $28 \mathrm{~cm}$ & $29 \mathrm{~cm}$ & $29 \mathrm{~cm}$ \\
\hline $\begin{array}{l}\text { Distância entre } \\
\text { os mamilos }\end{array}$ & $18 \mathrm{~cm}$ & $18 \mathrm{~cm}$ & $20 \mathrm{~cm}$ & $20 \mathrm{~cm}$ & $21 \mathrm{~cm}$ & $21 \mathrm{~cm}$ & $22 \mathrm{~cm}$ & $22 \mathrm{~cm}$ \\
\hline Raio do Seio & $6 \mathrm{~cm}$ & $7 \mathrm{~cm}$ & $7 \mathrm{~cm}$ & $8 \mathrm{~cm}$ & $9 \mathrm{~cm}$ & $9 \mathrm{~cm}$ & $10 \mathrm{~cm}$ & $10 \mathrm{~cm}$ \\
\hline $\begin{array}{l}\text { Altura da } \\
\text { cintura }\end{array}$ & $38 \mathrm{~cm}$ & $40 \mathrm{~cm}$ & $42 \mathrm{~cm}$ & $42 \mathrm{~cm}$ & $42 \mathrm{~cm}$ & $44 \mathrm{~cm}$ & $44 \mathrm{~cm}$ & $46 \mathrm{~cm}$ \\
\hline $\begin{array}{l}\text { Circunferência } \\
\text { da cintura }\end{array}$ & $76 \mathrm{~cm}$ & $80 \mathrm{~cm}$ & $84 \mathrm{~cm}$ & $88 \mathrm{~cm}$ & $92 \mathrm{~cm}$ & $96 \mathrm{~cm}$ & $100 \mathrm{~cm}$ & $102 \mathrm{~cm}$ \\
\hline $\begin{array}{l}\text { Circunferência } \\
\text { da barriga }\end{array}$ & $86 \mathrm{~cm}$ & $90 \mathrm{~cm}$ & $94 \mathrm{~cm}$ & $98 \mathrm{~cm}$ & $102 \mathrm{~cm}$ & $106 \mathrm{~cm}$ & $110 \mathrm{~cm}$ & $114 \mathrm{~cm}$ \\
\hline $\begin{array}{l}\text { Circunferência } \\
\text { do quadril }\end{array}$ & $94 \mathrm{~cm}$ & $96 \mathrm{~cm}$ & $100 \mathrm{~cm}$ & $104 \mathrm{~cm}$ & $108 \mathrm{~cm}$ & $114 \mathrm{~cm}$ & $120 \mathrm{~cm}$ & $126 \mathrm{~cm}$ \\
\hline
\end{tabular}

Dessa forma, pode-se considerar que as divergências entre as tabelas aqui apresentadas são parte da "[...] necessidade de combinar as medidas mínimas e as medidas máximas de uma população para se trabalhar com o denominado percentil" (DINIS; VASCONCELOS, 2014, p. 122). Vale ressaltar que os tipos corpóreos da população gestante brasileira devem ser levados em consideração para a construção de tabelas de medidas realmente eficazes para esse público.

Além disso, as tabelas propostas nessa pesquisa foram construídas com base nas mudanças corporais que acon- 
teceram no corpo da gestante até o $6^{\circ}$ mês de gestação; é nos três últimos meses da gestação que o volume da barriga se desenvolve significativamente e, por isso, seria necessário um estudo mais aprofundado quanto às circunferências e biótipos das gestantes.

Percebe-se que, em muitas gestantes, a medida de circunferência da barriga torna-se semelhante à circunferência do quadril. Com as medidas apresentadas nas tabelas propostas é possível desenvolver modelagens de peças destinadas ao tronco superior.

A seguir, o próximo tópico retoma os objetivos e explicita as considerações finais.

\section{CONSIDERAÇÕES FINAIS}

Esse artigo buscou propor um modelo de tabela de medidas que possa servir para o desenvolvimento de peças do vestuário para gestantes. O modelo de tabelas para gestante desenvolvido ao longo do presente artigo é teórico e seguiu diretrizes baseadas nas medidas dos manequins Draft, afinal, essa empresa faz estudos regulares sobre aferição de medidas corpóreas. As tabelas de medidas das literaturas aqui estudadas não condizem com a realidade vista na aferição de medidas realizada nessa pesquisa.

No decorrer desse artigo, e nas aferições de medidas corporais realizadas nas gestantes pesquisadas, utilizou-se o método manual com uso de fita métrica, seguindo diretrizes combinadas com os professores dos cursos do Instituto Federal de Santa Catarina campus Jaraguá do Sul/centro (SC). Essas medidas foram comparadas com as medidas dos manequins Drafts. Para realizar a tabela proposta, utilizou-se um padrão de das medidas de cintura, quadril e busto, que aumentaram de forma não abrupta, com vistas a atender o maior número de gestantes possível. Apesar de ser um modelo teórico de tabela gestacional, pode ser testada no futuro, principalmente para o desenvolvimento de peças que atendam ao público em período gestacional até o $6^{\circ}$ mês. 
Devido à pandemia do Covid-19 e a suspensão das aulas presenciais e decretos de quarentena não foi possível aferir e catalogar medidas de outras gestantes, afinal, um número maior de aferições ampliaria ainda mais a tabela teórica. Outra limitação encontrada foi a dificuldade de obter orientações em literaturas nacionais sobre como se realiza a aferição de medidas das circunferências da barriga e do quadril, entre outras medidas. A única orientação encontrada foi de Schimitt e Bataglion (2017), que oferecem diretrizes para as posições do corpo para a Educação Física e Fisioterapia, mas nada voltado ao segmento de vestuário.

Outra adversidade encontrada foram as tabelas de medidas para gestantes das lojas pesquisadas que, majoritariamente, não oferecem indicação alguma de qual período gestacional essas tabelas tratam - seja em meses, trimestres ou semanas -, o que dificultou delimitar e unir as informações. Essa seria uma indicação para a construção de futuras tabelas de medidas para gestantes.

Concluiu-se, também, que as medidas aferidas de gestantes do $1^{\circ}$ ao $6^{\circ}$ mês variam de acordo com seu tipo corpóreo, pois, por exemplo, podem aumentar entre dois e dez centímetros na circunferência do busto, o que pode ser notado tanto nas medidas das gestantes quanto dos manequins da marca Draft.

\section{REFERÊNCIAS}

ASSOCIAÇÃO BRASILEIRA DE NORMAS TÉCNICAS.

\section{Coletânea de Normas de Medidas do Corpo}

Humano. 2004. Disponível em: https://bit.

ly/3p9kSaS. Acesso em: 19 nov. 2020.

ASSOCIAÇÃO BRASILEIRA DE NORMAS TÉCNICAS. NBR

ISO 9241-171 de 09/2018. ERGONOMIA DA INTERAÇÃO

HUMANO-SISTEMA - PARTE 171: ORIENTAÇÕES

SOBRE ASSIBILIDADE DE SOFTWARE. 2018. Disponível

em: https://bit.ly/3EISRkZ. Acesso em: 5 nov. 2021. 
ASSOCIAÇÃO BRASILEIRA DE NORMAS TÉCNICAS. NBR

NBR ISO 7250-1 de 03/2010. MEDIDAS BÁSICAS DO

CORPO HUMANO PARA O PROJETO TÉCNICO — PARTE

1: DEFINIÇÕES DE MEDIDAS CORPORAIS E PONTOS

ANATÔMICOS. ABNT. Tabelas de Medidas. 2010. Disponível

em: https://bit.ly/3IXdS97. Acesso em: .26 jul. 2021.

ASSOCIAÇÃO BRASILEIRA DE NORMAS TÉCNICAS. NBR

13377: Medidas do corpo humano para vestuário:

padrões referenciais. Rio de Janeiro, ABNT, 1995.

ASTM INTERNACIONAL. Sobre ASTM Internacional.

ASTM, 2021. Disponível em: http://pt.astm.org/

ABOUT/about.html. Acesso em: 27 jul. 2021.

BOUERI, J. J. Antropometria Aplicado à Arquitetura,

Urbanismo e Desenho Industrial. $4^{\mathrm{a}}$ Ed. revisada.

São Paulo: E-book Editora das Letras, 2008.

BOUERI, J. J. Relatório Científico Final do projeto

de pesquisa: Antropometria Aplicada ao Projeto

e Dimensionamento do Vestuário Brasileiro.

Escola de Artes, Ciências e Humanidades da

Universidade de São Paulo - USP, 2010.

BROWNE, R. C. et al. Ergonomics Research Society.

British Medical Journal. 01(_):1009. 1949.

DINIS, P. M.; VASCONCELOS, A. F. C. Modelagem. In:

SABRÁ, F. G. C. Modelagem: Tecnologia em produção

de vestuário. 2 Ed. Rio de Janeiro: SENAI CETIQT;

São Paulo: Estação das Letras e Cores, 2014.

FLEURY, M. T. L.; WERLANG, S. R. da C. Pesquisa

aplicada: conceitos e abordagens. Rio de Janeiro:

Fundação Getúlio Vargas Sistema de Bibliotecas, 2016.

FRINGS, G. S. Moda: do conceito ao consumidor.

9. ed. Rio Grande do Sul: Bookman, 2012. 4 p. 
GERHARDT, T. E.; SILVEIRA, D. T. Métodos de pesquisa / [organizado por] Tatiana Engel Gerhardt e Denise Tolfo Silveira; coordenado pela Universidade Aberta do Brasil - UAB/UFRGS e pelo Curso de Graduação Tecnológica Planejamento e Gestão para o Desenvolvimento Rural da SEAD/UFRGS. Porto Alegre: Editora da UFRGS, 2009, 120 p.

\section{GIL, A. C. Como elaborar projetos de}

pesquisa. 4. ed. São Paulo: Atlas, 2008.

JONES, S. J. Fashion Design: manual do estilista. São Paulo: Cosac \& Naify, 2005.

KEHL, M.; BUCCl, E. Videologias: ensaio sobre televisão. São Paulo: Boitempo, 2004.

LEE, J. Y. et al. Comparison of body shape between USA and Korean women. International Journal of Clothing

Science and Technology. Vol. 19, No. 5, pp. 374-391, 2007.

LONGHI, T. C.; SANTOS, F. A. N. V. dos. Critérios ergonômicos no vestuário: uma proposta de sistematização de dados antropométricos. Dissertação de Mestrado, Florianópolis, curso de Pós-Graduação em Design, curso de Pós-Graduação em Design, UDESC. 2017. Disponível em: https:// bit.ly/3qbrW62. Acesso em: 6 ago. 2021.

MACHADO, G. A importância da modelagem no processo de venda do produto de moda para o público alvo gestante. Trabalho de Conclusão de Curso (Especialização em Modelagem do Vestuário), Universidade do Extremo Sul Catarinense, Criciúma, 2014, 80p. 
MARTINS, S. B. O conforto no vestuário: uma interpretação da ergonomia. Metodologia para avaliação de usabilidade e conforto no vestuário. 2005. 150 f. Tese (Doutorado)

- Curso de Pós-Graduação em Engenharia de Produção, Universidade Federal de Santa Catarina, Florianópolis, 2005. Disponível em: https://docplayer.com.br/41854502O-conforto-no-vestuario-uma-analise-da-relacao-entreconforto-e-moda.html. Acesso em: 17 ago. 2021.

MONTEIRO, G. C. P.; CARVALHO, A. P. L. de.; CANTELMO, E. M. Tabela de Medidas: Padronização ou idealização? CONTEXTMOD, [anais...], 2018. Disponível em: https://bit.ly/3FbSZo3 Acesso em: 23 jul. 2021.

OLIVETE, A. L. Tabela de medidas femininas: corpos e o que diz a ABNT. Audaces, 2020. Disponível em: https://audaces.com/tabela-de-medidasfemininas-2/. Acesso em: 2 ago. 2021.

RADICETTI, E. Medidas Antropométricas Padronizadas para a Indústria do Vestuário. Dissertação de mestrado, COPPE UFRJ. Rio de Janeiro, 1999.

SABRÁ, F. et al. Desenvolvendo a nova referência de medidas para o vestuário através da tecnologia de escaneamento de corpos 3D. In: $\mathbf{1 3}^{\circ}$ ERGODESIGN- USIHC, 2013. Disponível em: https://bit.ly/3siolG7. Acesso em: 15 jun. 2021.

SCHIMITT, B. D.; BATAGLION, G. A. Medidas e Avaliações

em Educação Física. 1. ed. rev. Brasil: Uniasselvi, 2017.

SILVEIRA, D. T.; CÓRDOVA, F. P. Pesquisa Científica. In: Métodos de pesquisa / [organizado por] Tatiana Engel Gerhardt e Denise Tolfo Silveira; coordenado pela Universidade Aberta do Brasil — UAB/UFRGS e pelo Curso de Graduação Tecnológica - Planejamento e Gestão para o Desenvolvimento Rural da SEAD/ UFRGS. Porto Alegre: Editora da UFRGS, 2009, 120 p. 
SILVEIRA, I. Modelo de gestão do conhecimento:

capacitação da modelagem de vestuário.

Lourdes Maria Puls (Org.). Florianópolis: Editora

UDESC, 2017. (Série Teses de Moda, v. 2).

SILVEIRA, I. Usabilidade do Vestuário: Fatores Técnicos/

Funcionais. Moda Palavra e-periódico, Florianópolis,

v. 1, n. 1, p. 21-29, jan./jun. 2008. Disponível em: https://

www.revistas.udesc.br/index.php/modapalavra/

article/view/7566/5070. Acesso em: 1 fev. 2021.

SOARES, L. D.; PETROSKI, E. L. Prevalência, Fatores

Etiológicos e Tratamento da Obesidade Infantil.

Revista Brasileira de Cineantropometria \&

Desempenho Humano, V 5, n 1, p. 63-74, 2003.

STEFANI, P. da S. Moda e Comunicação: a indumentária como forma de expressão. Juiz de Fora: UFJF, FACOM, 2. sem. 2005, 90 fl. mimeo. Projeto Experimental do Curso de Comunicação Social. Disponível em: http://www.ufjf.br/ facom/files/2013/04/PSilva.pdf. Acesso em: 12 mar. 2021.

THEIS, M. R. Criar, desenhar e modelar: o desenvolvimento de conteúdo interativo para aprendizagem nos processos de design de moda. Universidade da Região de Joinville, Joinville. 2018. 383 f.

WISNER, A. A análise da atividade em trabalhos complexos. In: Por dentro do trabalho: ergonomia, método e técnica. São Paulo: FTD, 1987. 